\title{
ARTICLE
}

Received 22 Aug 2014 | Accepted 17 Nov 2014 | Published 9 Jan $2015 \quad$ DOl: 10.1038/ncomms6878

\section{Magnetic control of transverse electric polarization in $\mathrm{BiFeO}_{3}$}

\author{
M. Tokunaga ${ }^{1}$, M. Akaki ${ }^{1}$, T. Ito ${ }^{2}$, S. Miyahara ${ }^{3}$, A. Miyake$^{1}$, H. Kuwahara ${ }^{4} \&$ N. Furukawa ${ }^{5}$
}

Numerous attempts have been made to realize crossed coupling between ferroelectricity and magnetism in multiferroic materials at room temperature. $\mathrm{BiFeO}_{3}$ is the most extensively studied multiferroic material that shows multiferroicity at temperatures significantly above room temperature. Here we present high-field experiments on high-quality mono-domain $\mathrm{BiFeO}_{3}$ crystals reveal substantial electric polarization orthogonal to the widely recognized one along the trigonal $c$ axis. This novel polarization appears to couple with the domains of the cycloidal spin order and, hence, can be controlled using magnetic fields. The transverse polarization shows the non-volatile memory effect at least up to $300 \mathrm{~K}$.

\footnotetext{
${ }^{1}$ Institute for Solid State Physics, University of Tokyo, 5-1-5 Kashiwanoha, Kashiwa, Chiba 277-8581, Japan. ${ }^{2}$ Electronics and Photonics Research Institute, National Institute of Advanced Industrial Science and Technology (AIST), Tsukuba, Ibaraki 305-8562, Japan. ${ }^{3}$ Department of Applied Physics, Fukuoka University, 8-19-1 Nanakuma, Jonan-ku, Fukuoka 814-0180, Japan. ${ }^{4}$ Department of Physics, Sophia University, 7-1 Kioi-cho, Chiyoda-ku, Tokyo 102-8554, Japan. ${ }^{5}$ Department of Physics and Mathematics, Aoyama Gakuin University, 5-10-1 Fuchinobe, Chuo-ku, Sagamihara, Kanagawa 229-8558, Japan. Correspondence and requests for materials should be addressed to M.T. (email: tokunaga@issp.u-tokyo.ac.jp).
} 
M agnetism and ferroelectricity are two representative subjects in condensed matter physics. The quest to understand their fundamentals has clarified how various materials can exhibit such properties, resulting in the development of various indispensable devises. However, these properties of solids are usually mutually exclusive in materials ${ }^{1}$. Although some exceptional materials, exhibiting both properties, have been recognized as ferroelectric magnets for over 40 years, the rich physics attributed to their cross-correlation had long remained unknown. The recent discovery of magnetic control of the electric polarization in manganite ${ }^{2}$ has attracted significant attention in pure and applied science. The following large number of studies revealed three major microscopic scenarios to induce the electric polarization by the spin system ${ }^{3}$ : spin current ${ }^{4-6}$, exchange striction ${ }^{7,8}$ and spin-dependent $p-d$ hybridization mechanisms $^{9-11}$.

The first scenario, called spin-current or inverse Dzyaloshinskii-Moriya mechanism, predicted the emergence of the electric polarization $(P)$ expressed by the vector spin chirality of the adjacent spins $\left(\mathbf{S}_{i, j}\right)$ and the unit vector connecting these sites $\left(\mathbf{e}_{i j}\right)$ as $\mathbf{P} \propto \mathbf{e}_{i j} \times\left(\mathbf{S}_{i} \times \mathbf{S}_{j}\right)$. This simple expression supported the excavation of multiferroic materials in which electric polarization can be controlled by external magnetic fields. The vector spin chirality in localized spin system is usually caused by the frustration between spin interactions. Inevitably, the magnetic ordering temperature is low in the frustrated spin systems. A large number of efforts have been devoted to realize hightemperature multiferroic and/or magnetoelectric (ME) materials $^{12-15}$, aiming to apply multiferroics to practical devices such as low-power-consumption memory devices. Although some of them exhibit significant ME effects at room temperature ${ }^{13-15}$, the electric polarization is absent or negligibly small at zero magnetic field and, hence, external magnetic fields are required to control the dielectric state.

$\mathrm{BiFeO}_{3}$ is unique, because it exhibits substantial multiferroicity well above room temperature ${ }^{16}$. It belongs to the trigonal $R 3 c$ space group (Fig. 1) below $\sim 1,100 \mathrm{~K}$, owing to the displacement of ions along and normal to the $\langle 111\rangle$ direction of the perovskite cubic unit ( $c$ axis of the trigonal cell) and the rotation of the ions around the same axis. Spontaneous polarization emerges along this axis with the magnitude exceeding $400,000 \mu \mathrm{C} \mathrm{m}^{-2}$ (ref. 17), which is superior to those of typical ferroelectric compounds such

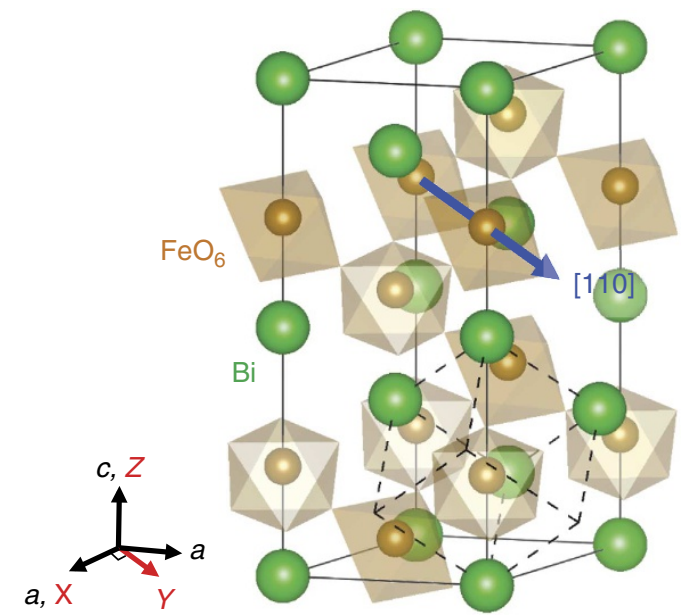

Figure 1 | Schematic crystal structure of $\mathbf{B i F e O}_{3}$. Thick blue arrow represents a direction of a spin modulation vector. Dashed lines represent the pseudo-cubic unit in the perovskite structure. $X, Y$ and $Z$ denote Cartesian coordinates. as $\mathrm{BaTiO}_{3}$. As $\mathrm{BiFeO}_{3}$ shows eight-fold degeneracy in the directions of the trigonal $c$ axis, its actual crystals often contain multiple crystallographic domains.

The spins of $\mathrm{Fe}^{3+}$ ions form a cycloidal order with the propagation vector pointing $\langle 110\rangle$ direction of the trigonal lattice below $\sim 640 \mathrm{~K}^{18-20}$. Such a non-collinear state can be explained by the Ginzburg-Landau theory, including the ME coupling term $^{21}$, or equivalently by the spin Hamiltonian, including Dzyaloshinskii-Moriya vectors normal to the $c$ axis $^{22,23}$. According to the spin-current mechanism, this cycloidal spin order involves $P$ along the $c$ axis. This magnetically induced component of $P$ can be removed at the transition from the cycloidal to the canted-antiferromagnetic state induced by a high magnetic field ${ }^{24,25}$, and indeed observed by Popov et al. ${ }^{24}$. Quantitative evaluation of the ME effect can be done only through the experiments on mono-domain crystals, otherwise the crystallographic domain boundaries, which are known to be conductive ${ }^{26}$, deteriorate the intrinsic signal. Recent high-field experiments on the mono-domain crystals showed the change in $P$ at the transition to be $\sim 210 \pm 30 \mu \mathrm{C} \mathrm{m}^{-2}$ below $400 \mathrm{~K}$ as a projected value on the cubic principal axis ${ }^{27}$. Details of the ME tensor remain open albeit indispensable to understand the multiferroicity of this material. In particular, we have to pay attention to the threefold degeneracy in the choice of the magnetic propagation vector that should affect the ME effects observed at low fields ${ }^{28}$.

In the present study, we performed experiments along the trigonal coordinates to obtain a fundamental understanding of the $\mathrm{ME}$ effects in $\mathrm{BiFeO}_{3}$. Recently developed high-quality $\mathrm{BiFeO}_{3}$ single crystals grown using the laser-diode heating floating-zone method $^{29}$ enabled these experiments. The results unexpectedly reveal the transverse (normal to the cycloidal spin rotation plane) component of the electric polarization $>800 \mu \mathrm{C} \mathrm{m}^{-2}$, which is controllable using magnetic fields. This novel component cannot be understood by the spin-current mechanism. However, on a bond with lower symmetry, the anti-symmetric pair of the adjacent spins can couple to $\mathbf{P}$ in a generic formula $P_{\alpha} \propto \sum_{\beta} d^{\alpha \beta}\left(\mathbf{S}_{i} \times \mathbf{S}_{j}\right)_{\beta}$, where $d$ is a tensor and non-zero components of it are determined by a local symmetry of the bonds ${ }^{30,31}$. In fact, all the tensor components of $d$ are allowed on nearest-neighbour bonds in $\mathrm{BiFeO}_{3}$, which induce the coupling between the transverse component of the electric polarization and cycloidal spin structure. As a result, we can reasonably reproduce the novel transverse component, which is confined to the magnetic domains and, hence, is easily controlled using a moderately high magnetic field even at $300 \mathrm{~K}$. Further, $\mathrm{BiFeO}_{3}$ shows the irreversible $\mathrm{ME}$ effect up to $300 \mathrm{~K}$, indicating its potential application in non-volatile memory devices.

\section{Results}

Magnetization and the longitudinal electric polarization. Figure 2a shows the magnetization $(M)$ curves for $\mathrm{BiFeO}_{3}$. Most of the data were measured at $4.2 \mathrm{~K}$, unless otherwise stated. Magnetic fields $(H)$ were applied along the $a$ axis, normal to the ca plane, and along the $c$ axis, which are defined as the $X, Y$ and $Z$ directions, respectively (Figs 1 and 2d). For the $H$ applied along the $X$ direction, the $M$ first slowly increased and then steeply increased at $\sim 18 \mathrm{~T}$. It then varied almost linearly with increasing $H$ (black line). Linearly extrapolating the high-field $M-H$ curve (dashed black line) gave a finite intercept of $0.048 \pm 0.002 \mu_{\mathrm{B}}$ per $\mathrm{Fe}$ at the zero-field, which is consistent with the emergence of the high-field canted antiferromagnetic phase ${ }^{25}$. The $H$ applied along the $Y$ direction (blue line) exhibited a trace almost identical to that of the $H$ applied along the $X$ direction. The difference between the blue and black traces is barely distinguishable in 
Fig. 2a. The $M$ for $H \| Z$, on the other hand, gradually increased in the low field, steeply increased with increasing $H$ between 22 and $26 \mathrm{~T}$, and then varied linearly with increasing $H$ up to $56 \mathrm{~T}$. Extrapolating the $M-H$ curve from the high-field phase to the zero-field indicated ferromagnetic components of $0.009 \pm 0.002 \mu_{\mathrm{B}}$ per $\mathrm{Fe}$, significantly lower than the trigonal plane ones, suggesting that the ferromagnetic moment was mostly confined to the trigonal plane.

Careful observation of the magnetization curve revealed hysteresis in the low-field region. Figure $2 \mathrm{~b}$ shows the steadyfield magnetization with increasing $H$ to $7 \mathrm{~T}$. The $M-H$ curve for the $H$ applied along the $Y$ direction was hysteretic, as shown by the thin blue line. The $M-H$ curve for the second field sweep coincided with the upper trace without showing hysteresis (solid blue symbols). The $M-H$ curve for the $H$ applied along the $X$ direction (not shown) showed similar hysteretic behaviour. The $M-H$ curve for the $H$ applied along the $Z$ direction, on the other hand, showed a reversible profile even for the first sweep (thin red line). We interpreted these hysteretic behaviours as the reorientation of the magnetic domains in $\mathrm{BiFeO}_{3}$.

Figure $2 \mathrm{c}$ shows the magnetic-field-induced change in the $P$ along the $Z$ direction $\left(\Delta P_{Z}\right)$. The effects of the $H$ applied along the $X$ and $Y$ directions are almost identical. The difference between the blue and black traces is barely identifiable. The $P_{Z}$ first gradually increased and then steeply increased at the magnetic transition with increasing $H$. The $P_{Z}$ continuously changed above the transition field. The $H$ applied along the $Z$ direction also caused $P_{Z}$ to first gradually increase then steeply increased at the transition. The low-order ME effect above the transition field showed the opposite sign to that of the in-plane fields. Although we could not determine the sign of the $\Delta P_{Z}$ with respect to the non-centrosymmetric crystal, the sign of the polarity relative to those of the different field directions is meaningful, because we changed the field direction while holding the wiring.
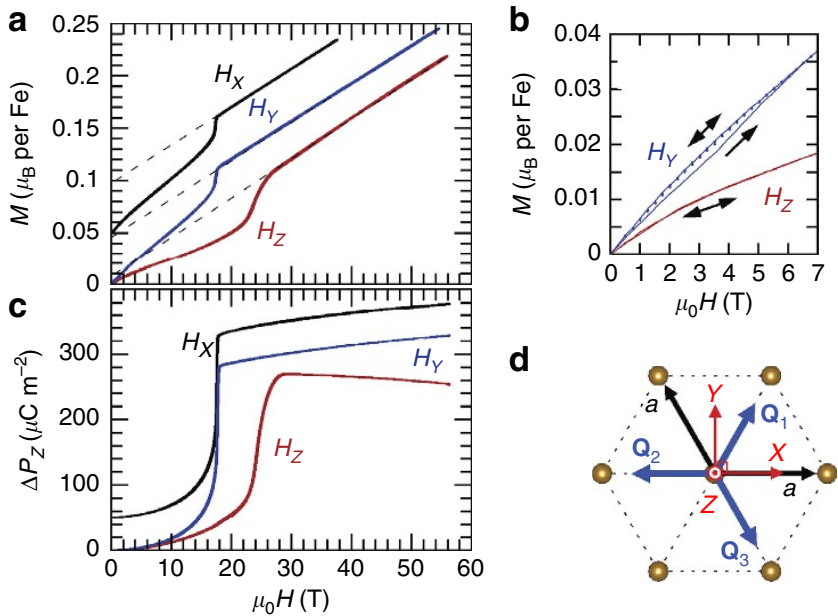

d

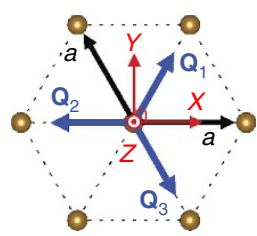

Figure 2 | Magnetic-field dependence of magnetization and electric polarization along the $\mathbf{Z}$ axis. (a) Magnetization curves for $\mathrm{BiFeO}_{3}$ measured at $4.2 \mathrm{~K}$ for $\mathrm{H}$ applied along $X$ (black), $Y$ (blue), and $Z$ (red) directions. The trace for $H_{X}$ was vertically offset by $0.05 \mu_{\mathrm{B}}$ per Fe for clarity. (b) Magnetization curves measured at $4.2 \mathrm{~K}$ up to $7 \mathrm{~T}$. Thin lines and solid symbols represent data measured during first and second field scans, respectively. (c) Magnetic-field-induced changes in polarization measured along $Z$ direction $\left(\Delta P_{Z}\right)$ for fields applied along $X, Y$ and $Z$ directions at $4.2 \mathrm{~K}$. The data for $H_{X}$ was vertically offset by $50 \mu \mathrm{Cm}^{-2}$ for clarity. (d) Schematic drawing of Cartesian coordinates $X, Y$ and $Z$. Thick blue arrows represent direction of modulation vectors for $\mathbf{Q}_{1}, \mathbf{Q}_{2}$ and $\mathbf{Q}_{3}$ magnetic domains.
Magnetic control of the transverse electric polarization. Figure $3 \mathrm{a}$ shows the change in the transverse component, $P_{Y}$, for the $H$ applied along the $X, Y$ and $Z$ directions. The thin black line shows that $P_{Y}$ steeply changed in the first trace for the $H \| X$ sweep at $\mu_{0} H \leq 7 \mathrm{~T}$. Here, $\mu_{0}$ is the space permeability. $P_{Y}$ then further increased $\sim 100 \mu \mathrm{C} \mathrm{m}^{-2}$ at the transition field and moderately changed with increasing $H$ up to $56 \mathrm{~T}$. $P_{Y}$ followed almost the same trace down to $10 \mathrm{~T}$ when the magnetic field was decreased, but did not significantly change in $\mu_{0} H \leq 7 \mathrm{~T}$. The second sweep (thick black line) traced the profile of the previous down-sweep without showing any prominent hysteresis. Applying $H_{Y}$ also produced an irreversible $\mathrm{P}-\mathrm{H}$ curve but in the opposite direction (thin blue line), while $\mu_{0} H_{Y} \leq 7 \mathrm{~T}$ during the virgin scan. Further, $\Delta P_{Y}$ at the transition field also showed the opposite sign from that for $H \| X$ and showed an almost linear ME effect with increasing $H$ to the highest field. The $P-H$ curve for $H_{Z}$, on the other hand, showed marginal hysteresis for $\mu_{0} H<20 \mathrm{~T}$, steeply increased when $\mu_{0} H \sim 26 \mathrm{~T}$ and then showed the linear ME effect. The observed $\Delta P_{Y}$ amounted to $500-800 \mu \mathrm{C} \mathrm{m}^{-2}$ and dominated the widely recognized $P_{Z}$ component caused by the cycloidal spin order (Fig. 1c).

Figure $3 \mathrm{~b}$ shows the $H$ dependence of $P_{X}$. The field-induced change in $P_{X}$ for $H_{X}$ and $H_{Y}$ is much smaller than those in $P_{Y}$ for the same, which remarkably contrasts with the almost isotropic magnetic properties observed in the magnetization measurements. Contrary to the small change in $P_{X}$ when the in-plane fields were applied, $P_{X}$ considerably changed only when $H_{Z}$ was applied. The solid red line in Fig. $3 \mathrm{~b}$ shows that the $\Delta P_{X}$ amounted to $800 \mu \mathrm{C} \mathrm{m}^{-2}$ at the transition field and then showed the linear ME effect when the field was higher. This considerable change, however, was less reproducible. The dashed red line represents the $P_{X}-H_{Z}$ profile measured for another piece of $\mathrm{BiFeO}_{3}$ crystal. The change measured at the transition became much smaller and showed the opposite sign of the previous one, whereas the high-field phase showed a similar linear ME effect in the same sign.

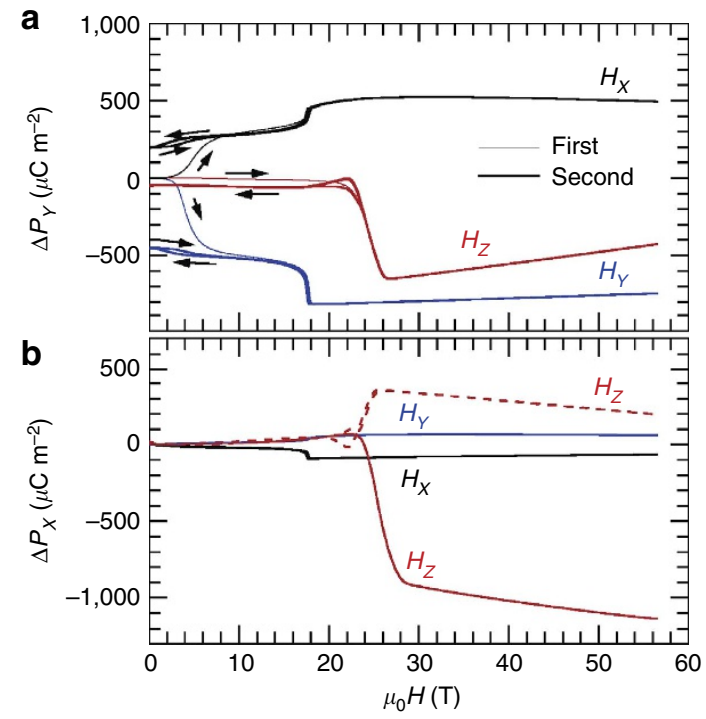

Figure 3 | Magnetic-field dependence of electric polarization along the $\boldsymbol{X}$ and $\boldsymbol{Y}$ axis. (a) Magnetic field dependence of $P_{Y}$ for $H$ applied along $X$ (black), $Y$ (blue) and $Z$ (red) directions. Thin and thick lines represent traces during first and second field scans, respectively. (b) Field-induced changes in $P_{X}$ for $H$ applied along all three directions. Dashed red line shows $\mathrm{P}_{X}-\mathrm{H}_{Z}$ curve measured for a different piece of $\mathrm{BiFeO}_{3}$ crystal. 


\section{Discussion}

The origin of the prominent transverse polarization could be antisymmetric spin pair-dependent electric polarization $P_{\alpha} \propto \sum_{\beta} d^{\alpha \beta}\left(\mathbf{S}_{i} \times \mathbf{S}_{j}\right)_{\beta}$, where $d^{\alpha \beta}$ is a coupling between spin and electric polarization ${ }^{30,31}$. For example, the cycloidal screw state with $\mathbf{Q}_{2}$ have finite $\left(\mathbf{S}_{i} \times \mathbf{S}_{i+x}\right)_{Y}\left[\left(\mathbf{S}_{i} \times \mathbf{S}_{i+y}\right)_{Y}\right]$ on bonds along $\langle 100\rangle(\langle 010\rangle)$ on a pseudo-cubic unit cell (Supplementary Fig. 1), which produces $P_{Y} \propto d^{Y Y}\left[\left(\mathbf{S}_{i} \times \mathbf{S}_{i+\chi}\right)_{Y}-\left(\mathbf{S}_{i} \times \mathbf{S}_{i+y}\right)_{Y}\right]$ in addition to the conventional term $P_{Z} \propto d^{Z Y}\left[\left(\mathbf{S}_{i} \times \mathbf{S}_{i+x}\right)_{Y}-\left(\mathbf{S}_{i} \times \mathbf{S}_{i+y}\right)_{Y}\right]$ due to the spin current mechanism. From the observed values of $\Delta P$ and the reported cycloidal spin structure ${ }^{20}$, the magnitudes of the coupling are estimated to be $\left|d^{Y Y}\right|=6.4 \times 10^{-32} \mathrm{C} \mathrm{m}$ and $\left|d^{Z Y}\right|=4.5 \times 10^{-32} \mathrm{C} \mathrm{m}$ as described in Supplementary Note 1 . The results indicate that the coupling of $P_{Y}$ is larger than that of $P_{Z}$. Owing to the symmetry of the crystal, the cycloidal screw states with $\mathbf{Q}_{1}, \mathbf{Q}_{2}$ and $\mathbf{Q}_{3}$ modulations can produce in-plane components of electric polarization $\left(P_{\mathrm{T}}\right)$ normal to the spin rotation plane, which is spanned by the $c$ axis and the spin modulation vector for each cycloidal domain (Fig. 4a,b). Applying magnetic fields is expected to favour the domains showing the spin rotation plane normal to the field. For example, applying $H_{Y}$ stabilizes the $\mathbf{Q}_{2}$ domain as illustrated in Fig. 4a. Here, $P_{X}=0$ and $P_{Y}=P_{\mathrm{T}}$ (Fig. 4a). Applying $H_{X}$, on the other hand, favours the $\mathbf{Q}_{1}$ and $\mathbf{Q}_{3}$ domains; hence, $P_{X} \sim 0$ if the $\mathrm{BiFeO}_{3}$ contains the same number of each domain, while $P_{Y}=-P_{\mathrm{T}} / 2$ regardless of the number of each domain (Fig. 4b). In this scenario, $P_{X}$ becomes small when the field is applied along the $X$ or $Y$ directions, as was experimentally observed, whereas it can become large when the field is applied at intermediate angles within the $X-Y$ plane. The response to applying $H_{Z}$ would be highly sensitive to possible field misalignment, as slight tilting towards a certain in-plane direction would determine the magnetic domains, that is, magnitude and direction of the $P_{\mathrm{T}}$, which are both consistent with the results shown in Fig. $3 b$.

a

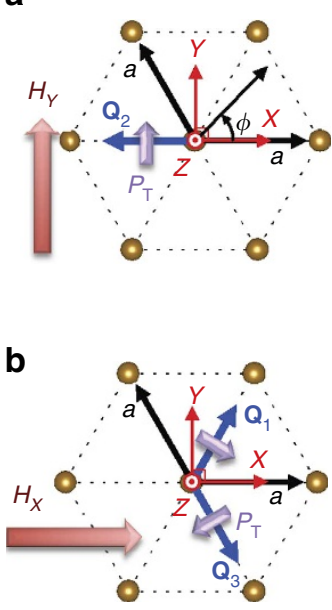

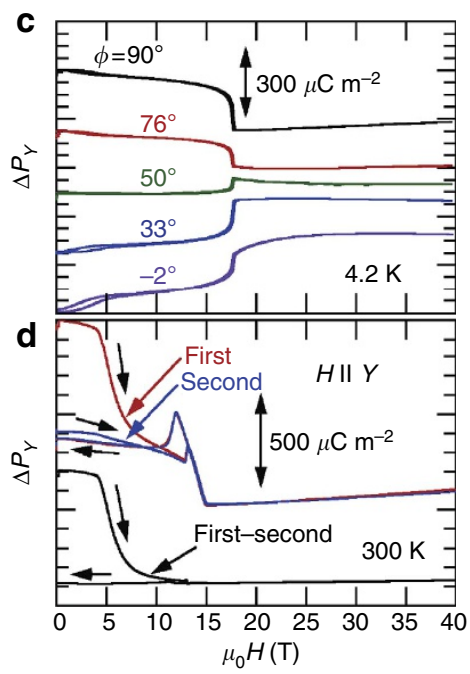

Figure 4 | Magnetic-field dependence of electric polarization along the $Y$ axis at various field direction and at temperature of $300 \mathrm{~K}$. Schematic drawings of magnetic field dependence of the magnetic domains for (a) $H \| Y$ and (b) $H \| X$. Short violet arrows represent direction of transverse electric polarization vectors $\left(P_{T}\right)$ for $\mathbf{Q}_{1}, \mathbf{Q}_{2}$ and $\mathbf{Q}_{3}$ magnetic domains. (c) Magnetic field dependence of $P_{Y}$ measured at various angles in range $-2^{\circ} \leq \phi \leq 90^{\circ}$. (d) $P_{Y}-H_{Y}$ curve measured at $300 \mathrm{~K}$. Red and blue lines represent traces for first and second field scans, respectively. Black line shows difference between them. Data in Fig. 4c, d were vertically offset for clarity.
To investigate these hypotheses, we studied the field dependence of $P_{Y}$ by changing the field direction within the $X-Y$ plane. Figure $4 \mathrm{c}$ shows $P_{Y}$ plotted as functions of the $H$ measured at various azimuthal angles, $\phi$, from the $X$ direction. The change in $P_{Y}$ at the transition field changed its sign between $50^{\circ}$ and $76^{\circ}$ with increasing the $\phi$, which is consistent with the expectation from our simple domain model in which the most stable domain changed from $\mathbf{Q}_{3}\left(P_{Y}=-P_{\mathrm{T}} / 2\right)$ to $\mathbf{Q}_{2}\left(P_{Y}=P_{\mathrm{T}}\right)$ at $\phi=60^{\circ}$.

The results so far suggest that a large transverse polarization was coupled with magnetic domains of $\mathrm{BiFeO}_{3}$. The irreversible magnetic and dielectric behaviour for $\mu_{0} H<10 \mathrm{~T}$ suggests that the re-orientation of the magnetic domains was preserved even after the $H$ was removed. To check whether the memory effect could be observed even at room temperature, we measured $P_{Y}-H_{Y}$ at $300 \mathrm{~K}$. As shown in Fig. $4 \mathrm{~d}, P$ irreversibly changed under the weak field only during the first sweep even at $300 \mathrm{~K}$. The difference between the first (red) and second (blue) profiles shows that the irreversible component of $P_{Y}$ amounted to $600 \mu \mathrm{C} \mathrm{m}^{-2}$ at $300 \mathrm{~K}$, as shown by the black line, which is almost comparable with or even larger than those measured at $4.2 \mathrm{~K}$. Further, $P$ steeply changed when the $\mu_{0} H$ was between 12 and $14 \mathrm{~T}$. Our $\mathrm{P}-\mathrm{H}$ measurements at various temperatures revealed that this anomaly is smoothly connected with the one measured at low temperature when $\mu_{0} H \sim 18 \mathrm{~T}$ (Supplementary Fig. 2 and Supplementary Note 2) and, hence, can be interpreted as the magnetic transition to the canted antiferromagnetic state.

Although our simple interpretation partly explains the observed change in the in-plane polarization, this domain model alone cannot explain the irreversible component measured at $\mu_{0} H<10 \mathrm{~T}$. According to this simple model, applying the $H$ along the $Y$ direction stabilized the $\mathbf{Q}_{2}$ domain, which would have the largest component of $P \| Y$. As shown in Fig. 4d, however, the initial point of the first sweep showed higher polarization than that of the second sweep, where we believe only the $\mathbf{Q}_{2}$ domain existed. The origin for this additional $P$ remains open at present. Judging from the magnetization and linear ME effect above $27 \mathrm{~T}$ (the red traces in Figs $2 a$ and $3 a$ ), the spin canting with the weak ferromagnetic moment of $0.1 \mu_{\mathrm{B}}$ per Fe along the $Z$ direction can induce $P_{Y} \sim 200 \mu \mathrm{C} \mathrm{m}^{-2}$. Possible emergence of weak ferromagnetic moment at the domain wall region ${ }^{32}$ might contribute somewhat to this extra component. Further systematic study will be necessary to solve this mystery.

Owing to the large electric polarization along the $c$ axis, that is, the corresponding lattice distortion, spins in $\mathrm{BiFeO}_{3}$ exhibit the cycloidal order. According to the generalized microscopic theory for anti-symmetric spin-dependent polarization ${ }^{30,31}$, this spin order involves parasitic spin-driven electric polarizations along with and also normal to the $c$ axis. In $\mathrm{BiFeO}_{3}$, the latter coupling $d^{Y Y}$ is larger than the former one $d^{Z Y}$ caused by the the spin current mechanism. This transverse component is confined to the magnetic domains and, hence, is easily controlled by $H$. The energy required for domain rotation can be estimated from the magnetization curve shown in Fig. $2 \mathrm{~b}$ as $\oint B d M=$ $1.18 \times 10^{3} \mathrm{~J} \mathrm{~m}^{-3}$ at $4.2 \mathrm{~K}$. From this energy density, we can expect that transverse polarization of $600 \mu \mathrm{C} \mathrm{m}^{-2}$ can be flopped by an electric field of $2 \mathrm{MVm}^{-1}$. This crude estimation reasonably agrees with the reported change in volume fraction of magnetic domains by electric field of $1.3 \mathrm{MV} \mathrm{m}^{-1}$ along the cubic axis ${ }^{33}$. Despite this small energy, the domain arrangement is preserved after removal of the $H$ even at $300 \mathrm{~K}$. These results indicate potential use of $\mathrm{BiFeO}_{3}$ as a three-state non-volatile memory corresponding to the three $\mathbf{Q}$ vectors $\mathbf{Q}_{1}, \mathbf{Q}_{2}$ and $\mathbf{Q}_{3}$.

In conclusion, we studied the magnetic field dependence of the electric polarization in $\mathrm{BiFeO}_{3}$ single crystals grown using the laser-diode heating floating-zone method. The electric polarization normal to the $c$ axis showed prominent field-induced change. 
The irreversible low-field polarization was memorized by the field sweep. Such non-volatile memory effect was also observed even at $300 \mathrm{~K}$, suggesting that the $\mathrm{BiFeO}_{3}$ can be useful for novel memory devices.

\section{Methods}

Experiments in high magnetic fields. Pulsed magnetic fields up to $56 \mathrm{~T}$ were generated by using pulse magnets installed at the Institute for Solid State Physics at the University of Tokyo. The magnetization was measured using the induction method in pulsed fields. We used a commercial magnetometer (MPMS; Quantum Design) for the magnetization measurements up to $7 \mathrm{~T}$, as shown in Fig. 2b. The field-induced changes in the electric polarization were calculated by numerically integrating the polarization currents ${ }^{34}$. To subtract the contribution of the voltage induced in the wiring, we averaged the signals measured during the positive and negative field sweeps (Supplementary Fig. 3 and Supplementary Note 3).

\section{References}

1. Hill, N. A. Why are there so few magnetic ferroelectrics. J. Phys. Chem. B 104, 6694 (2000).

2. Kimura, T. et al. Magnetic control of ferroelectric polarization. Nature 426, 55 (2003).

3. Tokura, Y., Seki, S. \& Nagaosa, N. Multiferroics of spin origin. Rep. Prog. Phys. 77, 076501 (2014)

4. Katsura, H., Nagaosa, N. \& Balatsky, A. V. Spin current and magnetoelectric effect in noncollinear magnets. Phys. Rev. Lett. 95, 057205 (2005).

5. Mostovoy, M. Ferroelectricity in spiral magnets. Phys. Rev. Lett. 96, 067601 (2006).

6. Sergienko, I. A. \& Dagotto, E. Role of the Dzyaloshinskii-Moriya interaction in multiferroic perovskites. Phys. Rev. B 73, 094434 (2006).

7. Choi, Y. J. et al. Ferroelectricity in an Ising chain magnet. Phys. Rev. Lett. 100, 047601 (2008)

8. Picozzi, S., Yamauchi, K., Sanyal, B., Sergienko, I. A. \& Dagotto, E. Dual nature of improper ferroelectricity in a magnetoelectric multiferroic. Phys. Rev. Lett. 99, 227201 (2007).

9. Jia, C., Onoda, S., Nagaosa, N. \& Han, J. H. Bond electronic polarization induced by spin. Phys. Rev. B 74, 224444 (2006).

10. Jia, C., Onoda, S., Nagaosa, N. \& Han, J. H. Microscopic theory of spinpolarization coupling in multiferroic transition metal oxides. Phys. Rev. B 76, 144424 (2007)

11. Arima, T. Ferroelectricity Induced by proper-screw type magnetic order. J. Phys. Soc. Jpn 76, 073702 (2007).

12. Kimura, T., Sekio, Y., Nakamura, H., Siegrist, T. \& Ramirez, A. P. Cupric oxide as an induced-multiferroic with high- $T_{c}$. Nat. Mater. 7, 291 (2008).

13. Kitagawa, Y. et al. Low-field magnetoelectric effect at room temperature. Nat. Mater. 9, 797 (2010).

14. Chun, S. H. et al. Electric field control of nonvolatile four-state magnetization at room temperature. Phys. Rev. Lett. 108, 177201 (2012).

15. Iyama, A. \& Kimura, T. Magnetoelectric hysteresis loops in $\mathrm{Cr}_{2} \mathrm{O}_{3}$ at room temperature. Phys. Rev. B 87, 180408(R) (2013).

16. Catalan, G. \& Scott, J. F. Physics and applications of bismuth ferrite. Adv. Mater. 21, 2463 (2009).

17. Shvartsman, V. V., Haumont, R. \& Kreisel, J. Large bulk polarization and regular domain structure in ceramic $\mathrm{BiFeO}_{3}$. Appl. Phys. Lett. 90, 172115 (2007).

18. Smolenskii, G. A. et al. New Ferroelectrics of complex composition. 4. Sov. Phys. Solid State 2, 2651 (1961).

19. Fischer, P., Polomska, M., Sosnowska, I. \& Szymański, M. Temperature dependence of the crystal and magnetic structures of $\mathrm{BiFeO}_{3}$. J. Phys. C 13, 1931 (1980)
20. Sosnowska, I., Peterlin-Neumaier, T. \& Steichele, E. Spiral magnetic ordering in bismuth ferrite. J. Phys. C 15, 4835 (1982).

21. Sosnowska, I. \& Zvezdin, A. K. Origin of the long period magnetic ordering in $\mathrm{BiFeO}_{3}$. J. Magn. Magn. Mater. 140-144, 167 (1995).

22. Jeong, J. et al. Spin wave measurements over the full brillouin zone of multiferroic $\mathrm{BiFeO}_{3}$. Phys. Rev. Lett. 108, 077202 (2012).

23. Zvezdin, A. K. \& Pyatakov, A. P. On the problem of coexistence of the weak ferromagnetism and the spin flexoelectricity in multiferroic bismuth ferrite. EPL 99, 57003 (2012).

24. Popov, Y. F. et al. Linear magnetoelectric effect and phase-transitions in $\mathrm{BiFeO}_{3}$. JETP Lett. 57, 69 (1993).

25. Ruette, B. et al. Magnetic-field-induced phase transition in $\mathrm{BiFeO}_{3}$ observed by high-field electron spin resonance: cycloidal to homogeneous spin order. Phys. Rev. B 69, 064114 (2004).

26. Seidel, J. et al. Conduction at domain walls in oxide multiferroics. Nat. Mater 8, 229 (2009)

27. Tokunaga, M., Azuma, M. \& Shimakawa, Y. High-field study of strong magnetoelectric coupling in single-domain crystals of $\mathrm{BiFeO}_{3}$. J. Phys. Soc. Jpn 76, 064713 (2010).

28. Tabares-Muñoz, C., Rivera, J. P., Bezinges, A., Monnier, A. \& Schmid, H. Measurement of the quadratic magneto electric effect on single crystalline $\mathrm{BiFeO}_{3}$. J. Appl. Phys. 24, 1051 (1985).

29. Ito, T., Ushiyama, T., Yanagisawa, Y., Kumai, R. \& Tomioka, Y. Growth of highly insulating bulk single crystals of multiferroic $\mathrm{BiFeO}_{3}$ and their inherent Internal strains in the domain-switching process. Cryst. Growth Des. 11, 5139 (2011).

30. Moriya, T. Theory of absorption and scattering of light by magnetic crystals. J. Appl. Phys. 39, 1042 (1968).

31. Kaplan, T. A. \& Mahanti, S. D. Canted-spin-caused electric dipoles: a local symmetry theory. Phys. Rev. B 83, 174432 (2011).

32. Darakychiev, M., Catalan, G. \& Scott, J. F. Landau theory od domain wall magnetoelectricity. Phys. Rev. B 81, 224118 (2010).

33. Lee, S., Ratcliff, II W., Cheong, S.-W. \& Kiryukhin, V. Electric field control of the magnetic state in $\mathrm{BiFeO}_{3}$ single crystals. Appl. Phys. Lett. 92, 192906 (2008).

34. Mitamura, H. et al. Dielectric polarization measurements on the antiferromagnetic triangular lattice system $\mathrm{CuFeO}_{2}$ in pulsed high magnetic fields. J. Phys. Soc. Jpn 76, 094709 (2007).

\section{Acknowledgements}

This work was supported by the MEXT of Japan Grants-in-Aid for Scientific Research 23340096, 25610087, 25800189 and 25287088) and the Mitsubishi Foundation.

\section{Author contributions}

M.T. planned and performed the experiments in collaboration with M.A. and A.M. Single crystals of $\mathrm{BiFeO}_{3}$ were grown by T.I. and prepared for experiments by M.A. and H.K. Theoretical calculations were done by S.M. and N.F. All authors discussed the results.

\section{Additional information}

Supplementary Information accompanies this paper at http://www.nature.com/ naturecommunications

Competing financial interests: The authors declare no competing financial interests

Reprints and permission information is available online at http://npg.nature.com/ reprintsandpermissions/

How to cite this article: Tokunaga, M. et al. Magnetic control of transverse electric polarization in $\mathrm{BiFeO}_{3}$. Nat. Commun. 6:5878 doi: 10.1038/ncomms6878 (2015). 